\title{
Isolation and identification of Moraxella cuniculi from a rabbit with keratoconjunctivitis
}

\author{
Dong-Kun Yang*, Ha-Hyun Kim, Jae-Young Yoo, Suk-Kyung Lim, Soon-Seek Yoon, In-Soo Cho \\ Animal and Plant Quarantine Agency, Ministry of Agriculture, Food and Rural Affairs, Gimcheon 39660, Korea,
}

(Received: July 3, 2017; Revised: August 1, 2017; Accepted: August 10, 2017)

\begin{abstract}
A Gram-negative, catalase- and oxidase-positive, coccus-shaped bacterium was isolated from a rabbit with keratoconjunctivitis. Colonies of the isolate were round, smooth, and exhibited hemolytic activity on $5 \%$ sheep blood agar. Scanning electron microscopy revealed 0.4 to $0.5 \mu \mathrm{m}$ diameter oval cocci. Partial 16S rRNA gene (1446 bp) sequence analysis demonstrated the isolate had significant homology with the Moraxella cuniculi CCUG2154 strain isolated from a rabbit in Germany in 1973. Our isolate was designated as APQAB1701. Antibiotic susceptibility tests demonstrated that APQAB1701 was sensitive to 24 antibiotics; 3 of the antibiotics (nalidixic acid, spectinomycin, and colistin) had minimal inhibitory concentrations $\geq 32 \mu \mathrm{g} / \mathrm{mL}$ against the isolate.
\end{abstract}

Keywords: Moraxella cuniculi, identification, isolation, rabbits

The genus Moraxella is a Gram-negative bacterium that causes opportunistic infection in humans and animals [6]. Morphologically, Moraxella species are short rods or coccobacilli, or in the case of Moraxella (M.) catarrhalis, diplococci. In humans, M. catarrhalis is the best-known Moraxella spp. [7]. M. lacunata and M. osloensis have been reported to cause blepharoconjunctivitis and hematological malignancies in humans $[3,6]$. In veterinary medicine, M. bovis is the causative agent of infectious bovine keratoconjunctivitis, which is characterized by conjunctivitis, keratitis, corneal opacity, and ulceration, a clinical syndrome called pinkeye $[4,10]$. As a strict aerobe, M. bovis is confined to the cornea and conjunctiva, resulting in a progressive, non-self-limiting keratitis, ulceration and, ultimately, rupture of the cornea. The disease is relatively common, infecting cattle worldwide, although there have been no reports of $M$. bovis or $M$. spp among domestic animals in South Korea. In this study, we isolated and identified M. cuniculi from a rabbit with clinical keratoconjunctivitis and named the isolate APQAB1701. This study investigated the biological/morphological characteristics and antibiotic resistance of the APQAB1701 isolate.

A disease associated with conjunctivitis and congestion of the eyelid was reported in a 1-year-old rabbit raised on a farm in Gyeongsang Province, Korea, in 2017. The farm raising about 200 rabbits had morbidity rate of 1 to $2 \%$, and the mortality rate was close to zero. The farm had one rabbit with conjunctivitis and hyperplasia of the eyelid. Ten swab samples from three rabbits were collected from the rabbit farm. Three swab samples were collected from around eyelid of the infected rabbit and other seven samples were also collected from two rabbits showing wasting disease. All swab samples were cultured on blood and chocolate agar plates to isolate bacteria. The plates were incubated for $24 \mathrm{~h}$ at $37^{\circ} \mathrm{C}$. The colonies were passed three times to obtain pure colonies and tested using Gram staining and catalase and oxidase tests. The morphology of the isolate was observed using electron microscopy. Bacteria enriched in brain heart infusion broth were fixed with $2 \%$ glutaraldehyde overnight, and with $1 \%$ osmium tetroxide in phosphate-buffered saline for $2 \mathrm{~h}$. The fixed bacteria were coated with gold ions and then observed under a JSM/LV-6460 electron microscope (JEOL, Japan).

Genomic DNA was extracted from the isolated colonies using a genomic DNA extraction kit (iNtRON Biotechnology, Korea), according to the manufacturer's instructions. PCR was used to amplify the $16 \mathrm{~S}$ rRNA gene using the forward primer 5'-AGA GTT TGA TCM TGG CTC AG-3' and reverse primer 5'-TAC CGY TAC CTT GTT ACG ACT T-3'. The sequencing reactions were performed in a DNA Engine Tetrad 2 Peltier Thermal Cycler (Bio-Rad Laboratories, USA) using the ABI BigDye(R) Terminator v3.1 Cycle Sequencing Kit (Applied Biosystems, USA), following the manufacturer's protocols. Single-pass sequencing was performed with each template using sequencing primers (forward 5'-GGA TTA GAT ACC CTG GTA-3', reverse 5'-CCG TCA ATT CMT TTR AGT TT-3'). The fluorescent-labeled fragments were purified using the method recommended by Applied

*Corresponding author

Tel: +82-54-912-0785, Fax: +82-54-912-0812

E-mail: yangdk@korea.kr 

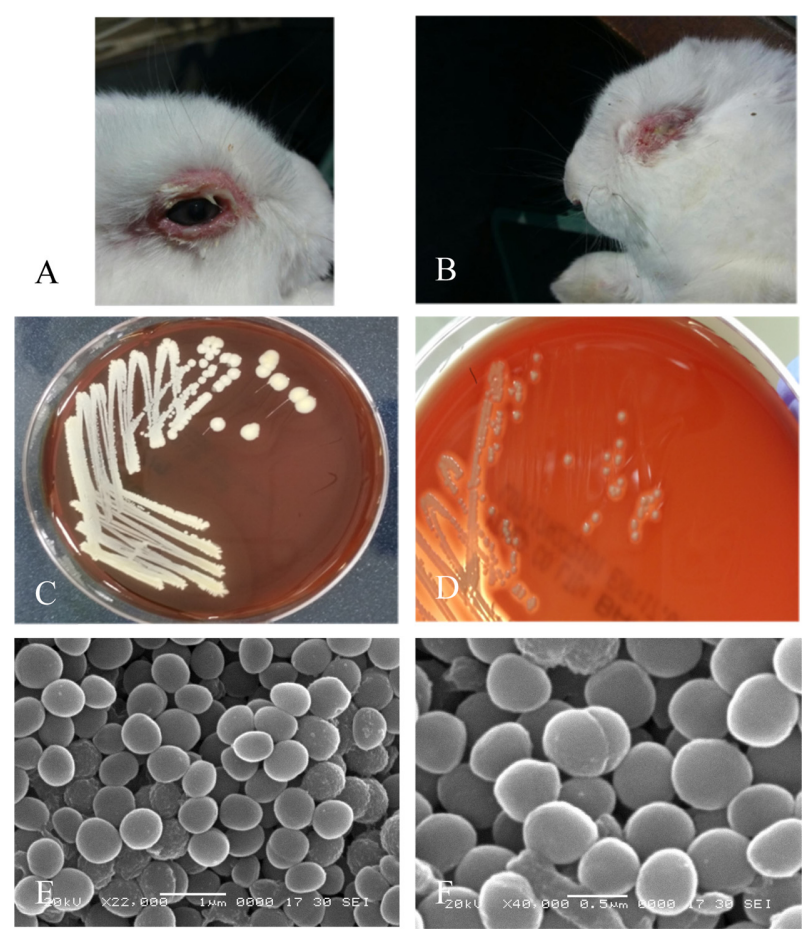

Fig. 1. One eye of the rabbit showed severe keratoconjunctivitis and hyperplasia (A), and the other eye was blinded by inflammation (B). Pure isolation of Moraxella cuniculi isolated on chocolate agar (C); the isolate was hemolytic on a $5 \%$ sheep blood agar plate (D). Morphology of the Moraxella cuniculi APQAB1701 isolated on scanning electron microscopy. The bacteria were oval and measured 0.4 to $0.5 \mu \mathrm{m}$ in diameter (E and F).

Biosystems, as it removes the unincorporated terminators and dNTPs. The samples were subject to electrophoresis in an ABI 3730xl DNA Analyzer (Applied Biosystems). The nucleotide sequences and accession numbers of the strains used for the phylogenetic analysis were obtained from the GenBank database (National Center for Biotechnology Information, USA). The partial nucleotide sequence of the $16 \mathrm{~S}$ rRNA genes obtained from the isolate was compared with those of known bacteria using Clustal W 2 [2]. Genetic distances were calculated using the Kimura 2-parameter model, and a phylogenetic tree was constructed using the neighborjoining method with 1,000 bootstrap replicates in MEGA6 software [8].

To evaluate antibiotic resistance, minimal inhibitory concentrations (MICs) were determined on KRNV4F and BOPOGF MIC plates (Trek Diagnostic Systems, USA) using the standard broth dilution methods described in the Clinical and Laboratory Standard Institute guidelines for the following 27 antibiotics: amoxicillin/clavulanic acid, ampicillin, penicillin, cefoxitin, ceftiofur, cephalothin, clindamycin, ciprofloxacin, danofloxacin, enrofloxacin, nalidixic acid, colistin, florfenicol, chloramphenicol, gentamicin, neomycin, streptomycin, spectinomycin, tetracycline, chlortetracycline, oxytetracycline, trimethoprim/sulfamethoxazole, sulfadimethoxine, tiamulin, tilmi-
Table 1. Results of antibiotic resistance of Moraxella cuniculi, APQAB1701

\begin{tabular}{lclc}
\hline \hline \multicolumn{1}{c}{ Antibiotics } & MIC $(\mu \mathrm{g} / \mathrm{mL})$ & \multicolumn{1}{c}{ Antibiotics } & MIC $(\mu \mathrm{g} / \mathrm{mL})$ \\
\hline Amoxicillin & $<2$ & Gentamicin & $<1$ \\
Ampicillin & $<0.25$ & Neomycin & $<2$ \\
Penicillin & $<0.12$ & Streptomycin & 8 \\
Cefoxitin & 4 & Spectinomycin & 64 \\
Ceftiofur & 1 & Tetracycline & $<2$ \\
Cephalothin & $<2$ & Chlortetracycline & $<0.5$ \\
Clindamycin & $<0.25$ & Oxytetracycline & $<0.5$ \\
Ciprofloxacin & $<0.12$ & Trimethoprim & $<0.12$ \\
Danofloxacin & $<0.12$ & Sulfadimethoxine & $<256$ \\
Enrofloxacin & $<0.12$ & Tiamulin & 1 \\
Nalidixic Acid & 32 & Tilmicosin & $<4$ \\
Colistin & $>32$ & Tulathromycin & 8 \\
Florfenicol & 4 & Tylosin & 2 \\
Chloramphenicol & 8 & & \\
\hline
\end{tabular}

MIC, minimum inhibition concentration.

cosin, tulathromycin, and tylosin.

Figure 1 shows the rabbit with severe conjunctivitis and hyperplasia around the eyelid. Three pure colonies were isolated from four samples obtained from the rabbit cultured on blood and chocolate agar plates. The nucleotide sequences of the three colonies identified them as M. cuniculi, Pasteurella multocida, and Corynebacterium spp. We named the $M$. cuniculi isolate APQAB1701 and investigated its biochemical characteristics and morphological features. The isolate was Gram-negative, catalase-positive, oxidase-positive, and aerobic. Colonies of the isolate were circular, smooth, and hemolytic on 5\% sheep blood agar (Fig. 2). Morphologically, the isolate consisted of oval cocci measuring 0.4 to $0.5 \mu \mathrm{m}$ in diameter on electron microscopy (Fig. 1). A 1,446-nucleotide partial 16S rRNA gene sequence was determined for the APQAB1701 isolate and compared with partial 16S rRNA gene sequences of 35 other bacteria obtained from GenBank. The nucleotide sequence analysis showed that the APQAB1701 isolate shared identical homology with the CCUG2154 strain, which was isolated from a rabbit in Germany in 1973 , and had $97 \%$ similarity with M. bovis (accession No. AF005183) (Fig. 2). The antimicrobial susceptibility of the isolate was tested with standard broth dilution methods. The isolate was sensitive to 24 antibiotics, and 3 antibiotics (nalidixic acid, spectinomycin, and colistin) had MICs $\geq 32 \mu \mathrm{g} / \mathrm{mL}$ against the isolate (Table 1).

Most Moraxella species except M. bovis are considered to be part of the normal mucosal flora of humans and animals. The Moraxella species implicated in a variety of conditions in humans and domestic animals are frequently found in mixed bacterial or viral infections [1, 9]. In our study, $M$. cuniculi was isolated with P. multocida, and Corynebacterium sp., indicating that the bacterium was an opportunistic 


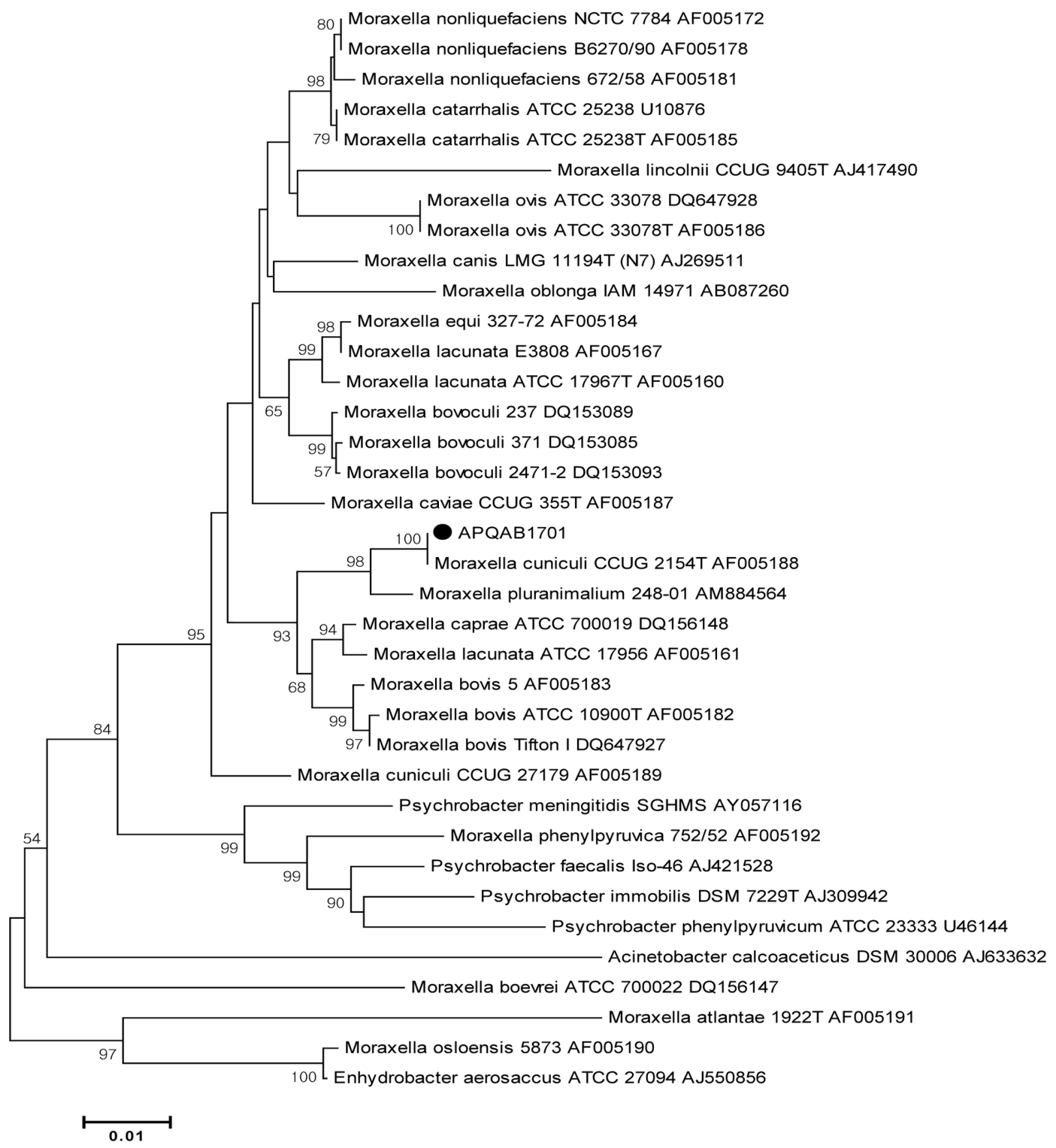

Fig. 2. Phylogenetic tree based on 1446 bp of the $16 \mathrm{~S}$ rRNA gene. The isolate, APQAB1701, had a $100 \%$ similarity with CCUG2154T which was isolated from a German rabbit in 1973.

pathogen associated with eye disease. Several Moraxella species are reported to hemolyze sheep blood, including $M$. bovis, M. canis, M. caprae, M. caviae, and M. boviae, but not M. cuniculi $[6,9]$. In our study, the M. cuniculi, APQAB1701 isolate showed hemolytic activity on $5 \%$ sheep blood agar, indicating that M. cuniculi produces a hemolysin and should be considered pathogenic. Furthermore, it is necessary to identify genes encoding potential virulence factors, such as phospholipases, outer membrane protein and proteolytic enzymes [5]. The 16S rRNA sequence analysis of bacteria is a reliable method that can be applied for species identification $[6,9]$. The pure isolate, APQAB1701 was confirmed to be $M$. cuniculi, as it showed $100 \%$ homology with the CCUG2154 strain isolated from a rabbit in Germany in 1973. Regarding treatment, the $M$. cuniculi isolate described in this study was susceptible to 24 antibiotics, and the rabbit infected with mixed bacteria containing $M$. cuniculi will be treated after performing antibiotic susceptibility tests on the collected samples.

In conclusion, we report the identification of $M$. cuniculi isolated from a Korean rabbit. These findings indicated that M. cuniculi had hemolytic activity in relation to conjunctivitis and hyperplasia of the eyelid in a rabbit. This study did not demonstrate the virulence of $M$. cuniculi. It is therefore necessary that future studies investigate the virulence of the isolate in normal rabbit.

\section{Acknowledgments}

This work was supported financially by a grant (N1543083- 
2014-18-01) from the Animal and Plant Quarantine Agency, Ministry of Agriculture, Food and Rural Affairs, Republic of Korea.

\section{References}

1. Farajzadah Sheikh A, Saki N, Roointan M, Ranjbar R, Yadyad MJ, Kaydani A, Aslani S, Babaei M, Goodarzi H. Identification of Alloiococcus otitidis, Streptococcus pneumoniae, Moraxella catarrhalis and Haemophilus influenzae in children with otitis media with effusion. Jundishapur $\mathbf{J}$ Microbiol 2015, 8, e17985.

2. Larkin MA, Blackshields G, Brown NP, Chenna R, McGettigan PA, McWilliam H, Valentin F, Wallace IM, Wilm A, Lopez R, Thompson JD, Gibson TJ, Higgins DG. Clustal $\mathrm{W}$ and Clustal $\mathrm{X}$ version 2.0. Bioinformatics 2007, 23, 2947-2948.

3. Maayan H, Cohen-Poradosu R, Halperin E, Rudensky B, Schlesinger Y, Yinnon AM, Raveh D. Infective endocarditis due to Moraxella lacunata: report of 4 patients and review of published cases of Moraxella endocarditis. Scand J Infect Dis 2004, 36, 878-881.

4. Pugh GW Jr, McDonald TJ. Identification of bovine carriers of Moraxella bovis by comparative cultural examinations of ocular and nasal secretions. Am J Vet Res 1986, 47, 23432345.
5. Sosa V, Umpiérrez A, Acquistapace S, Zunino P. Virulence genes in Moraxella spp. isolates from infectious bovine keratoconjunctivitis cases. J Infect Dev Ctries 2015, 9, 10281032.

6. Sung JY, Hong SK, Kim EC. The first Korean case of Moraxella osloensis bacteremia in a patient with acute myeloid leukemia. Ann Lab Med 2014, 34, 256-258.

7. Tamang MD, Dey S, Makaju RK, Jha BK, Shivananda PG, Bhramadatan KN. Prevalence of Moraxella catarrhalis infections of the lower respiratory tract in elderly patients. Kathmandu Univ Med J (KUMJ) 2005, 3, 39-44.

8. Tamura K, Dudley J, Nei M, Kumar S. MEGA4: Molecular Evolutionary Genetics Analysis (MEGA) software version 4.0. Mol Biol Evol 2007, 24, 1596-1599.

9. Vela AI, Arroyo E, Aragón V, Sánchez-Porro C, Latre MV, Cerdà-Cuéllar M, Ventosa A, Domínguez L, FernándezGarayzábal JF. Moraxella pluranimalium sp. nov., isolated from animal specimens. Int J Syst Evol Microbiol 2009, 59, 671-674.

10. Yeruham I, Perl S, Elad D. Infectious bovine keratoconjunctivitis and lymphofollicular hyperplasia of the third eyelid in heifers. J Vet Med B Infect Dis Vet Public Health 2001, 48, 137-141. 